\title{
DEVELOPMENT OF AN ASYMMETRIC POWER DIVIDER FOR A HIGH- POWER RF DISTRIBUTION SYSTEM AT KEK
}

\author{
Takeshi Takahashi, Shogo Sakanaka, Masaaki Izawa, Institute of Materials Structure Science, \\ High Energy Accelerator Research Organization (KEK), Tsukuba, Ibaraki 305-0801, Japan
}

\begin{abstract}
We have developed an $\mathrm{rf}$ power divider which is used for an $\mathrm{rf}$ distribution system in high-energy electron storage rings. This divider has four waveguide ports, and it can split a high $\mathrm{rf}$ power by a ratio of $1: 2$ at an operating frequency of about $508.6 \mathrm{MHz}$. Other properties are very similar to those of a magic-T. By combining this divider with a conventional magic- $\mathrm{T}$, one can design an $\mathrm{rf}$ distribution system in which three cavities are driven by a single klystron. The design procedure of the power divider, a low-power measurement, and a high-power test result are described.
\end{abstract}

\section{INTRODUCTION}

In most of the storage rings or synchrotrons, the number of rf cavities, which are driven by a single highpower tube, is chosen to be one, two, four, or eight. This is because an rf power is usually divided by either a magic- $\mathrm{T}$ or a $3-\mathrm{dB}$ coupler. In some storage rings, it is more desirable to drive three or six cavities by a single tube due to an available space in their straight section or to other reasons. However, such a system has rarely been constructed because there is no conventional rf divider which can split the rf power into three while having desirable isolation between the cavities.

One of such applications is a possible cavity layout for the $6.5-\mathrm{GeV}$ synchrotron light source "PF-AR" at KEK. The PF-AR is under a large-scale reconstruction [1], which is aimed at longer beam lifetime and higher beam currents. In the near future, a new undulator is planned to be installed in a west straight section, which is currently occupied by four 11-cell cavities. As one of the possible plans, we consider to install three cavities in each west and east straight section. For this purpose, we need to drive three cavities by a single klystron.

We have developed an asymmetric power divider [2,3] for this application. This divider can split a high $\mathrm{rf}$ power by a ratio of $1: 2$. Other properties, such as isolations between the ports, are very similar to those of a magic-T. Combining this divider with a conventional magic-T, one can split an rf power into three equal ones.

\section{DESIGN}

We consider a four-port device, which can split an incident rf from port 1 to both ports of 2 and 3. For our application, the following characteristics are required: (i) all of the ports should be matched well; (ii) in order to avoid unwanted coupling between the cavities, the ports 2 and 3 should be isolated to each other; and (iii) to obtain the power ratio of $1: 2,\left|S_{21}\right|^{2}=1 / 3$ and $\left|S_{31}\right|^{2}=2 / 3$, where $\mathrm{S}_{\mathrm{ij}}$ are the S-parameters. We designed a power divider, having such properties, using a computer code "HighFrequency Structure Simulator (HFSS)".

A designed structure is shown in Fig. 1. This structure was optimized at an operating frequency of $508.58 \mathrm{MHz}$ for the PF-AR. It has four WR1500 waveguide ports. A post (diameter, $40 \mathrm{~mm}$; height, $333 \mathrm{~mm}$ ), a rod (diameter, $51 \mathrm{~mm}$ ) and an iris in this structure are used to adjust the rf characteristics. An incident TE10 wave from port 1 is split into two TE10 waves, and goes out from ports 2 and 3 by a power ratio of 1:2.

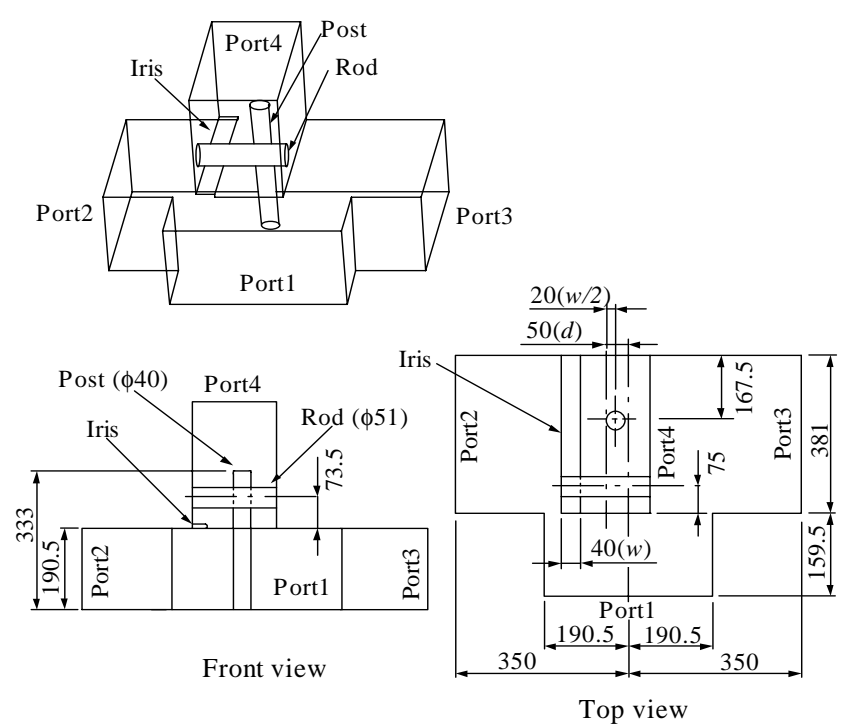

Figure 1: Optimized design of the asymmetric rf-power divider

The rf characteristics were optimized by the following procedures:

(1) Adjust the horizontal position ( $d$ in Fig. 1) of port 4 so that the desired power-split ratio is obtained. The post is moved together. At this step, an undesirable transmission $\mathrm{S} 41$ from port 1 to port 4 increases.

(2) S41 can be reduced by attaching an iris to the origin of port 4 and then by optimizing the width ( $w$ in Fig. 1 ) of the iris. The horizontal position ( $w / 2$ in Fig. 1) of the post was adjusted dependently so that the post would be located at the middle of the aperture of the iris; this gave good isolation between port 1 and port 4 .

(3) Port 1 can be matched well by adjusting the longitudinal (back or forth) position of the post. 
(4) Port 4 can be matched well by adjusting the position of the rod.

By applying the above procedure repeatedly, we could optimize the rf characteristics. Figure 2 shows the changes in the S-parameters in the above step (1). It shows that the ratio of S21: S31 can be controlled almost arbitrary by adjusting the horizontal position $(d)$ of port 4 . Note that when $d$ is positive, port 4 is shifted towards port 2.
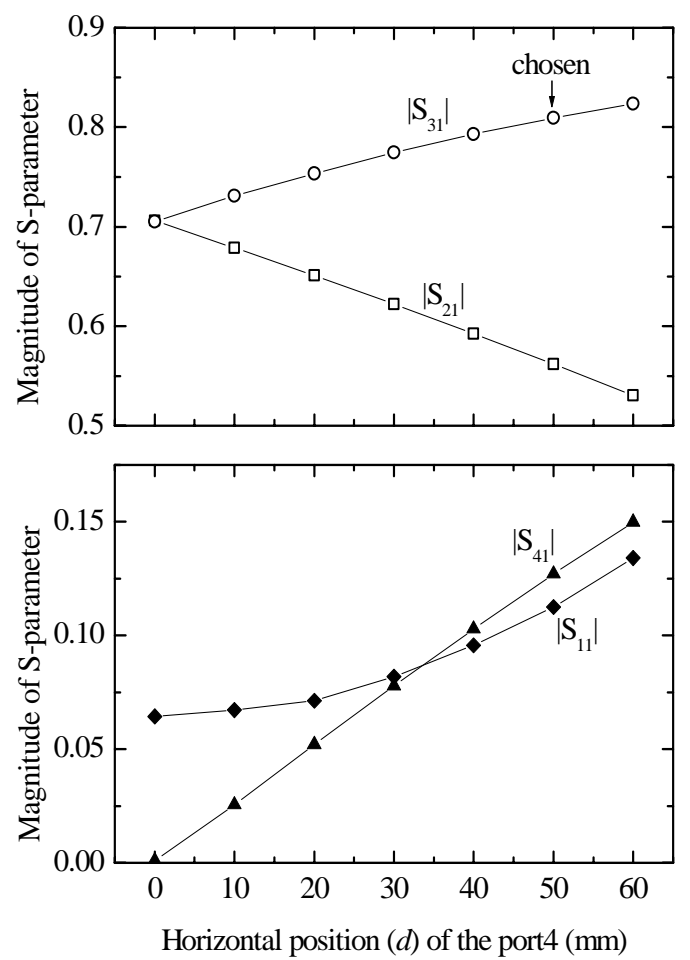

Figure 2: Dependence of the S-parameters on the horizontal position $(d)$ of the port 4 , which was obtained from the HFSS simulation.

As a result of the above optimization, we obtained the final design (shown in Fig. 1) having the following Smatrix:

$S_{c}=\left[\begin{array}{cccc}0.048 \angle 20^{\circ} & 0.564 \angle 148^{\circ} & 0.824 \angle 129^{\circ} & 0.024 \angle 159^{\circ} \\ 0.564 \angle 148^{\circ} & 0.061 \angle 110^{\circ} & 0.013 \angle 98^{\circ} & 0.823 \angle-37^{\circ} \\ 0.824 \angle 129^{\circ} & 0.013 \angle 98^{\circ} & 0.029 \angle 32^{\circ} & 0.566 \angle 124^{\circ} \\ 0.024 \angle 159^{\circ} & 0.823 \angle-37^{\circ} & 0.566 \angle 124^{\circ} & 0.038 \angle 8^{\circ}\end{array}\right]$.

Here, $S_{c}$ is the S-matrix obtained from a HFSS calculation at a frequency of $508.6 \mathrm{MHz}$. Each matrix element is represented by its magnitude followed by its phase in degrees. The above $\mathrm{S}$-matrix is very close to our target. For example, the designed values of $\mathrm{S} 21$ and $\mathrm{S} 31$ are about 0.564 and 0.824 , respectively, while the target values for them are about 0.577 and 0.816 , respectively. All of the ports are matched well, and the undesirable transmissions S41 and S32 are sufficiently small. Although there is some phase difference between the split waves from port 1 to ports 2 and 3, this phase difference can easily be compensated by adding some delay line through the transmission line. Note that it is also possible to design another power divider having an arbitrary power-split ratio by the same procedure as above.

\section{LOW POWER MEASUREMENT}

Based on the above-mentioned design, we made a high-power test model of the asymmetric power divider. The main body of the power divider was constructed by welding some plates of aluminum alloy. The post and the rod were fixed by screws. The tip of the post was rounded by $10 \mathrm{~mm}$ in radius. An (EIAJ-standard) FUDR6 waveguide flange was welded to each WR1500 waveguide port. The length of each waveguide port was designed to be the same as that of an existing magic-T so that this divider would be mechanically compatible with the magic-T. Figure 3 shows a photograph of the completed power divider.

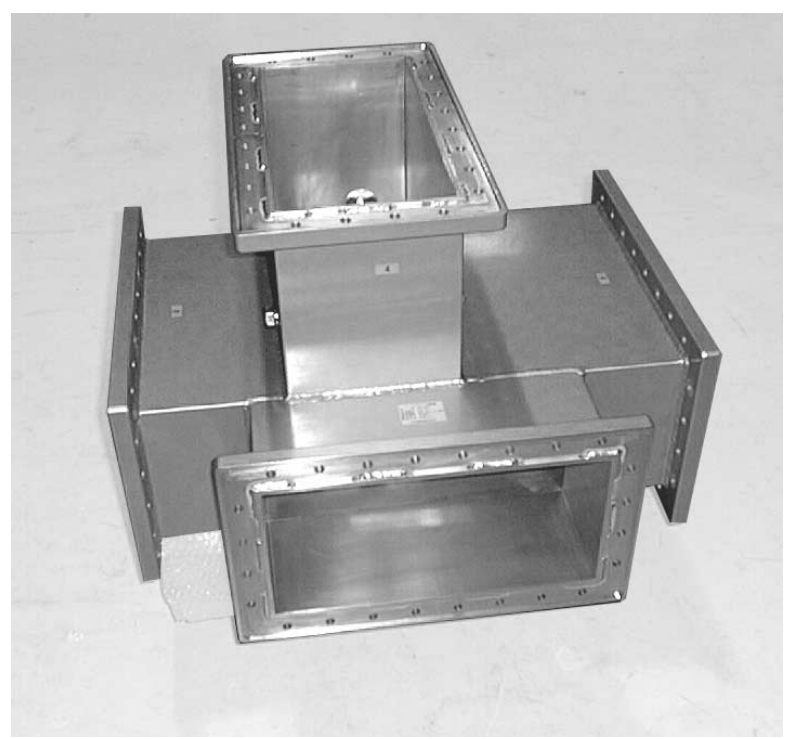

Figure 3: A high-power test model of the asymmetric power divider.

We carried out a low-power measurement on this prototype using a Network Analyzer. In the measurement, two coaxial lines from the Network Analyzer were connected to two of the power-divider ports through coaxial-waveguide transformers. The other two ports of the divider were terminated by other transformers and $50 \Omega$-terminators. In order to measure the rf characteristics accurately, we applied the Thru-Reflect-Line (TRL) calibration technique before the measurement. After the calibration, the measurement planes were taken at the entrances of the divider ports. Then, the measured data directly gave the S-parameters of the power divider without any additional phase factors. Measurement errors would mainly come from slight mismatches (VSWR: 1.086 and 1.016) of the transformers that were used as the terminators for the remaining ports. We obtained the following S-matrix at a frequency of $508.6 \mathrm{MHz}$ : 
$S_{m}=\left[\begin{array}{cccc}0.066 \angle \approx 10^{\circ} & 0.570 \angle 151^{\circ} & 0.820 \angle 127 & 0.014 \angle-1^{\circ} \\ 0.570 \angle 151^{\circ} & 0.055 \angle \approx 160^{\circ} & 0.046 \angle-10^{\circ} & 0.820 \angle-31^{\circ} \\ 0.82 \measuredangle 127 & 0.046 \angle-10^{\circ} & 0.056 \angle \approx 30^{\circ} & 0.568 \angle 126^{\circ} \\ 0.014 \angle-1^{\circ} & 0.820 \angle-31^{\circ} & 0.569 \angle 126 & 0.08 \measuredangle \approx-50^{\circ}\end{array}\right]$,

Here, the symbol $\approx$ in $S_{m}$ denotes relatively less-accurate data, which were checked by the consistency between the different measurements. The measured S-matrix $\left(S_{m}\right)$ agreed fairly well with the designed one $\left(S_{c}\right)$. Concerning the major S-parameters $\mathrm{S} 21, \mathrm{~S} 31, \mathrm{~S} 42$, and $\mathrm{S} 43$, the measured and designed ones agree within about $1 \%$ for their magnitudes, and about 6 degrees in their phases. For the other S-parameters having small magnitudes, relatively large differences in their phases possibly came from a slight mismatch at the remaining two ports or from a slight construction error; however, they are not important for our use. We also measured frequency responses of the S-parameters of this divider, which showed the desired rf characteristics within a sufficiently wide frequency range of $10 \mathrm{MHz}$.

\section{HIGH POWER TEST}

We tested the prototype power divider under high power. Figure 4 shows a block diagram of the high-power test station, which is usually used for the KEK B-factory (KEKB). A high rf power at a frequency of $508.9 \mathrm{MHz}$ was provided by a 1-MW klystron (E3786 from Toshiba corp.) through a 1-MW circulator.

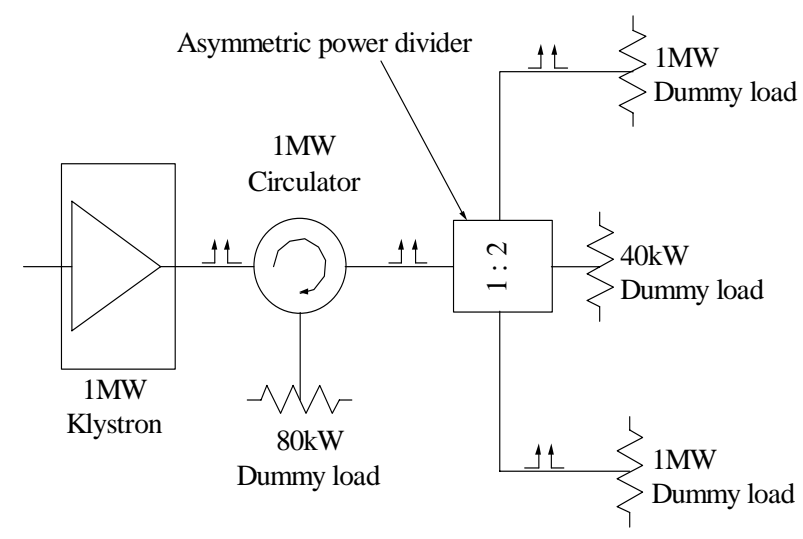

Figure 4: Setup for the high-power test.

The input $\mathrm{rf}$ was divided by the asymmetric power divider, and the split powers were dissipated in two 1MW dummy loads. The fourth port of the divider was terminated by a $40-\mathrm{kW}$ dummy load. The incident power and the reflected power were measured using directional couplers.
We successfully tested the power divider up to an input power of about $800 \mathrm{~kW}$ in the continuous-wave $(\mathrm{CW})$ mode. At the maximum power of $802 \mathrm{~kW}$, which was kept for about 10 minutes, the reflected power from the divider was about $1.4 \mathrm{~kW}$. The output powers from the port 2 and from the port 3 were then about $271 \mathrm{~kW}$ and $541 \mathrm{~kW}$, respectively, which were estimated from the temperature rises of the cooling water for dummy loads. Noting that those estimated powers were not very accurate due to some fluctuation in the temperature, the monitored output powers are consistent with the low-power measurement result. We also monitored the temperatures of the power divider at several locations. Under the maximum power, the temperatures of the outer wall were about $48-60^{\circ} \mathrm{C}$, which was comparable to that of a straight waveguide. The highest temperature of about $73^{\circ} \mathrm{C}$ occurred at the fixing bolt for the post. We experienced no trips due to reflected power. After the test, we checked the inside walls of the power divider, and found no sign of discharges.

\section{CONCLUSIONS}

The asymmetric power divider, which is used to split the input rf power by a ratio of $1: 2$, has been developed. The design procedure of this divider was established using computer simulations. A prototype power-divider was fabricated based on this design; it showed rf characteristics which were very similar to those obtained from the simulation. We have demonstrated that this prototype divider can be operated under a high power of up to $800 \mathrm{~kW}$ in the $\mathrm{CW}$ mode without any problems. This power divider will be useful in constructing an $\mathrm{rf}$ distribution system, in which a single klystron drives three accelerating cavities.

\section{ACKNOWLEDGMENTS}

We would like to thank S. Isagawa, H. Nakanishi and K. Ebihara for their cooperation with this work.

\section{REFERENCES}

[1] "Design Report on Advanced Ring for Pulse X-rays (PF-AR) ”, KEK Report 97-2, 1997 [in Japanese].

[2] T. Takahashi et al., "Design of rf components for the Advanced Ring at KEK", APAC'98, Tsukuba, Japan, 1998, pp. 761-763.

[3] T. Takahashi et al., "Development of an asymmetric power divider for a high-power RF distribution system", to be published in IEEE Trans. Nucl. Sci. 